\title{
Photopolarization of Fucus zygotes is determined by time sensitive vectorial addition of environmental cues during axis amplification
}

\author{
Kenny A. Bogaert ${ }^{1}$, Tom Beeckman ${ }^{2,3}$ and Olivier De Clerck ${ }^{1 *}$ \\ ${ }^{1}$ Research Group Phycology, Biology Department, Ghent University, Ghent, Belgium \\ 2 Department of Plant Biotechnology and Bioinformatics, Ghent University, Ghent, Belgium \\ ${ }^{3}$ Department of Plant Systems Biology, Vlaams Instituut voor Biotechnologie, Ghent, Belgium
}

\section{Edited by:}

Benedicte Charrier, Centre National de la Recherche Scientifique, France

Reviewed by:

David Smyth, Monash University, Australia

Darryl L. Kropf, University of Utah, USA

Isabel Bäurle, University of

Potsdam, Germany

*Correspondence:

Olivier De Clerck, Research Group Phycology, Biology Department, Ghent University, Krijgslaan 281 S8, 9000 Ghent, Belgium e-mail: olivier.declerck@ugent.be
Fucoid zygotes have been extensively used to study cell polarization and asymmetrical cell division. Fertilized eggs are responsive to different environmental cues (e.g., light, gravity) for a long period before the polarity is fixed and the cells germinate accordingly. First, it is commonly believed that the direction and sense of the polarization vector are established simultaneously as indicated by the formation of an F-actin patch. Secondly, upon reorientation of the zygote, a new polar gradient is formed and it is assumed that the position of the future rhizoid pole is only influenced by the latter. Here we tested these two hypotheses investigating photopolarization in Fucus zygotes by reorienting zygotes $90^{\circ}$ relative to a unilateral light source at different time points during the first cell cycle. We conclude that fixation of direction and sense of the polarization vector is indeed established simultaneously. However, the experiments yielded a distribution of polarization axes that cannot be explained if only the last environmental cue is supposed to determine the polarization axis. We conclude that our observations, together with published findings, can only be explained by assuming imprinting of the different polarization vectors and their integration as a vectorial sum at the moment of axis fixation. This way cells will average different serially perceived cues resulting in a polarization vector representative of the dynamic intertidal environment, instead of betting exclusively on the perceived vector at the moment of axis fixation.

Keywords: Fucus, polarization, asymmetrical cell division, positional information, Brown algae, intrinsic factors, embryogenesis, patterning

\section{INTRODUCTION}

Patterning of an embryo is often dependent on maternally determined polarity. In most organisms at least one axis is established during oogenesis while the cell is still enclosed in parental tissue. For example, the apical-basal pattern of egg cells in land plants is maternally determined (Ueda and Laux, 2012). Fucoid zygotes are exceptions to this general pattern. The eggs are radially symmetric the moment they are released in the water column and are therefore especially interesting model systems for the study of the establishment of cell polarity (Quatrano and Shaw, 1997; Kropf et al., 1999).

Cell polarization of fucoid eggs starts directly after fertilization resulting invariably in an asymmetric cell division. The establishment of polarity is a continuum of overlapping events that are traditionally subdivided in two stages: axis selection and axis amplification (Kropf, 1997). The polarization vector is specified during the process of axis selection. The future rhizoid pole is initially specified by the sperm entry site that co-localizes with the cortical location of an F-actin site (Hable and Kropf, 2000). This axis serves as the default pathway for polarization of zygotes cultured in the absence of environmental signals. Subsequently, the zygote develops an extracellular adhesive matrix and attaches to the substrate (Vreeland et al., 1993). At this stage, environmental cues can still override the weaker default axis provided by the sperm entry site. Unidirectional light, for example, induces the rhizoids to grow from the shaded side (Hurd, 1920). The F-actin patch at the sperm entry site disassembles and a new F-actin patch is formed at the nascent rhizoid pole in accordance with the new vector (Alessa and Kropf, 1999; Kropf et al., 1999). After axis selection, there is a period of axis amplification involving localized secretion. The endomembrane system, organized by the F-actin and microtubular cytoskeleton, accumulates adhesive material preferentially at the future rhizoid pole, determining an intracellular polar axis that will be termed here the "axis amplification vector" (Vreeland et al., 1993; Hadley et al., 2006; Hable et al., 2008; Peters and Kropf, 2010). The onset of this stage is likely controlled by a developmental clock as it starts a couple of hours after fertilization (AF) (Alessa and Kropf, 1999), probably in order to avoid axis amplification before the zygote has had the chance to settle using the freshly acquired adhesive layer.

Although in intertidal habitats environmental cues are likely to be much more diverse as well as dynamic, unilateral light is the most commonly used cue for in vitro polarization experiments. Zygotes are plated in petridishes or on coverslips and illuminated 
laterally throughout the cell cycle. In the intertidal, a number of different vectors are perceived at once and these signals are integrated together (Hable, 2014, this issue). Until shortly before germination the axis remains, surprisingly, labile and susceptible to realignment to a new vector (Alessa and Kropf, 1999). The axis becomes fixed as a consequence of the local secretion of Golgiderived material including sulfated fucan (F2) into the cell wall (Hogsett and Quatrano, 1978; Shaw and Quatrano, 1996b) and the establishment of the "axis stabilizing complex" (Fowler and Quatrano, 1995; Belanger and Quatrano, 2000). In case the environmental conditions change and a new environmental vector is perceived during the photoresponsive period, a new rhizoid site will be selected according to this new vector and amplified (Kropf et al., 1999). It is only prior to germination that the polarization axis becomes permanently fixed (Fowler and Quatrano, 1995; Belanger and Quatrano, 2000).

The new axis amplification vector is not established by mere rotation of the old one but is established de novo because of two reasons. (i) First, it has been found that polarized light induces zygotes to develop two rhizoids at opposite poles (Jaffe, 1958). (ii) Secondly, several factors such as the F-actin patch (Alessa and Kropf, 1999), polar secretion (Schröter, 1978), the dihydropyridine receptors (Shaw and Quatrano, 1996a), ionic currents and cortical clearing (Nuccitelli, 1978) show a reoriented polar organization quickly after reorientation as predicted by the new light vector. Moreover, cells with two F-actin patches in the short time frame after the reorientation have been observed (Alessa and Kropf, 1999).

It is assumed that the axis stabilizing vector is the fixed form of the last axis amplification vector and therefore the future rhizoid pole is identical to the new shaded hemisphere. Despite some remnants of the first environmental vector such as the polar adhesive (Schröter, 1978), the first light vector is thought to have no influence on the polarization axis. Interestingly, the new axis is assembled and amplified rapidly as it does not need additional time (Kropf et al., 1999). To our knowledge, there is no evidence for the assumed link between the de novo established amplification vector and the axis stabilization vector. Most reorientation experiments reorient only at one time point at the beginning of the photoresponsive period, leaving only a very short time for the initial amplification vector to leave a putative detectable influence on the final polarization axis. Secondly, the reorientation experiments use $180^{\circ}$ changes. Cells that grow a rhizoid according to the first light vector can be either interpreted as being fixed before reorientation or having a larger influence of the first light cue than the second. Therefore, these experiments cannot exclude the possibility that the old axis amplification vector influences the final axis stabilization vector. Only Schröter (1978) used a ca. $125^{\circ}$ reorientation at the beginning of the photoresponsive period, but reported detailed results of a single zygote only.

When the zygotes are reoriented in relation to unilateral light the axis amplification vector changes. The orientation of the polarization axis and the position of the rhizoid pole are implicitly assumed to change in one step. The observation of negative photopolarization (with the zygote developing the rhizoid at the lighted side instead of the dark side) under treatments that alter intracellular $\mathrm{Ca}^{2+}$ gradients (Robinson, 1996), may suggest that fixation of direction and sense involves two separate steps in fucoid cell polarization (Fowler and Quatrano, 1997). However, this has never been tested due to the complete reliance on $180^{\circ}$ reorientation experiments (Cove, 2000). Such reorientation changes only the sense of the incoming light vector and not its direction, while reorientations with $90^{\circ}$ change both sense and direction of environmental vector and therefore make it possible to test whether sense and direction become fixed with the same kinetics. Here we tested two implicit assumptions: (i) that the polarization process cannot be separated on a temporal scale into a two step process (ii) that the last 'axis amplifying vector' is identical to the 'axis stabilizing vector'. Our results indicate that it is indeed impossible to separate the alignment of the direction and sense of the polarization vector into a two-step process. Our $90^{\circ}$ reorientation experiments, however, contradict the hypothesis that only the last axis amplifying vector defines the axis stabilizing vector (and polarization axis), thereby offering some novel insights to longstanding views regarding Fucus polarity establishment.

\section{METHODS AND MATERIALS CULTURE}

Sexually mature receptacles of the fucoid alga Fucus spiralis Linnaeus were collected near Wimereux (France), Oostende (Belgium) and Blankenberge (Belgium) and stored at $4^{\circ} \mathrm{C}$ until use. Release was induced by rinsing the receptacles with tap water and subsequently placing them in natural daylight at room temperature in natural filtered seawater. The time of fertilization was considered at $1 \mathrm{~h}$ after exposure to daylight. Debris was filtered out using a $100 \mu \mathrm{m}$ nylon mesh. Zygotes were plated on poly-L-coated coverslips and grown at ca. $16^{\circ} \mathrm{C}$. For reorientation experiments, coverslips were carefully placed on petridishes (2-3 replicate coverslips per petridish) at $3 \mathrm{~h}$ after fertilization. Petridishes were exposed to cool white unidirectional fluorescent light at ca. $60 \mu \mathrm{mol}$ photons $\mathrm{m}^{-2} \mathrm{~s}^{-1}$ on black sheets of paper to avoid reflection. For each replicate experiment a $90^{\circ}$ or $180^{\circ}$ reorientation of one petridish was executed at time points varying between 7 and $21 \mathrm{~h} \mathrm{AF}$. Each petridish underwent exactly one reorientation event. $48 \mathrm{~h}$ after fertilization, the orientation of the polarization vector was determined by scoring the zygotes with rhizoids in $45^{\circ}$ intervals with the direction of the light vectors in the middle of an interval as illustrated in Figure 1. The first light vector illuminated the zygotes at an arbitrarily chosen angle of $0^{\circ}$, the second at an angle of $90^{\circ}$ or $180^{\circ}$.

\section{TBO STAINING}

Polarization of Fucus zygotes was assayed by Toluidine Blue O staining (TBO), which stains sulfated fucoidin indicative of polar secretion of Golgi-derived material into the cell wall (Quatrano and Crayton, 1973; Quatrano and Shaw, 1997). Fucus zygotes were stained for $15 \mathrm{~min}$ with $0.1 \%$ Toluidine Blue O ASW at $\mathrm{pH}$ 1.5. Slides were rinsed in $99 \%$ ethanol three times for ca. $5 \mathrm{~min}$ and once for $1 \mathrm{~h}$ before being mounted in tap water.

\section{STATISTICS}

Sample sizes for each replicate experiment are listed in Supplementary Tables 1-3 together with the raw data. Second 


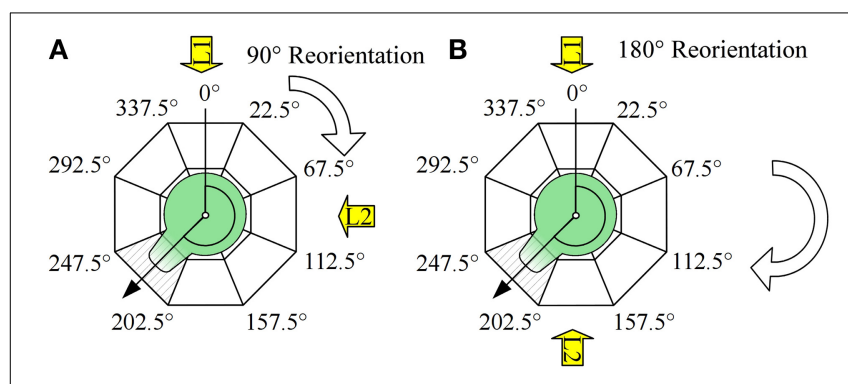

FIGURE 1 | Experimental design and scoring method and (A) $90^{\circ}$ reorientation, (B) $180^{\circ}$ reorientations. Angle between the vector pointing toward the first direction of the light (black line at $0^{\circ}$ ) and the polarization vector (arrow, determined by the rhizoid outgrowth and the center of the cell) was scored by estimating its orientation on a angular scale with a $45^{\circ}$ resolution as depicted (dashed interval). Numbers depict borders of the intervals.

order mean angles of the polarization vector for the three samples under $90^{\circ}$ degrees were calculated per replicate assuming a unimodal circular distribution using the procedure of Batschelet (1978). As a measure of angular concentration the $r$-value (Batschelet, 1972) was used. Significance of the mean angles was established by a testing procedure due to Hotelling (Hotelling, 1931). Parameters of a gompertz sigmoid functions were fitted through the mean values using $\mathrm{R}$ (version 3.1.0) and the package $n l s$ (Non-linear Least Squares).

\section{RESULTS}

Fucus zygotes were reoriented $90^{\circ}$ in relation to the unidirectional light source in a clockwise direction. Angles of the polarization vector with the light source (L1) were scored and represented as radial graphs for each time point of reorientation (Figure 2). Mean angles and a measure of concentration are depicted as the angle described by and the length of the arrow, respectively. All mean angles of the polarization vector were statistically significant (Hotelling test, $P<0.05$ ).

The mean angle of polarization decreases gradually from $270^{\circ}$ to $180^{\circ}$ with increased illumination time of L1 (Figure 3). For example with the reorientation carried out at $7 \mathrm{~h} \mathrm{AF}$ almost all zygotes fixed their polarization vector in accordance with L2, while a reorientation at $18 \mathrm{~h}$ AF produced a population of rhizoids pointing in the direction and sense described by the L1 environmental vector. During intermediate time points no enrichment of zygotes with polarization direction aligned to L1 but with misaligned sense of polarity (relative to L1) can be observed. Second order mean angles were plotted over time together with a regressed Gompertz sigmoid function (Figure 3). The angle gradually turns from an angle that is in accordance with the second light vector $\left(270^{\circ}\right)$ to one in accordance with the first light vector $\left(180^{\circ}\right)$ with increasing illumination time by the first light vector. Twelve hours AF an intermediate angle of $225^{\circ}$ is obtained and at $15 \mathrm{~h} 30 \mathrm{AF}$, the angle is very close to $180^{\circ}$.

In a separate experiment Fucus zygotes were reoriented $180^{\circ}$ at $10.5,12,13,14.5$, and $16.5 \mathrm{~h} \mathrm{AF}$. One hundred and eighty degrees reorientations at $10.5 \mathrm{~h}$ and $12 \mathrm{~h} \mathrm{AF}$ produced a diametrically bimodal circular distribution (Figure 4). For example, at an intermediate time point of a reorientation at $12 \mathrm{~h} \mathrm{AF,} 33 \pm 2.71 \%$ of the zygotes polarized in the interval determined by L2, 47.67 $\pm 2.88 \%$ polarized in the interval determined by L1, while only $19.33 \pm 2.28 \%$ polarize in one of the other six intervals (mean \pm standard deviation) (Figure 4). The percentage of zygotes that fix a polarization vector on their shaded side in respect of L1 is plotted over time (Figure 5, squares), which results in a very similar curve like the one that describes the reorientation of the angle at $90^{\circ}$. Under $180^{\circ}$ reorientation $50 \%$ of zygotes are polarized according to $\mathrm{L} 1$ at about $11.5 \mathrm{~h} \mathrm{AF}$, while a $90^{\circ}$ reorientation results in a polarization axis of $45^{\circ}$ with respect to L1 and L2 around the same time point.

To determine the timing of the establishment of the axis stabilizing vector the polarized secretion of Golgi-derived material was monitored in populations of zygotes grown in unilateral light using TBO staining as a marker. The percentage of zygotes with asymmetric deposition of F2 fucoidin as assayed by TBO staining is plotted over time (Figure 5, triangles, dotted line) and reaches ca. $50 \%$ only at about $13.5 \mathrm{~h} \mathrm{AF}$.

\section{DISCUSSION}

Currently it is accepted that Fucus zygotes in the process of polarization commit to the last applied environmental vector before axis fixation (Bisgrove, 2007; Hable and Hart, 2010). Our data cannot be explained by the currently accepted scenario. Different possible scenarios for the resultant rhizoid orientation are detailed below.

Under the currently accepted model, whereby reorientation of polarizing zygotes prior to axis fixation results in de novo establishment of a new axis amplification vector (Figure 6, Situation I), we can expect a mixture of embryos with rhizoids fixed according L1 or L2 light vector. A $90^{\circ}$ reorientation would result in a bimodal distribution of zygotes polarized at an angle $90^{\circ}$ relative to each other. Under a $180^{\circ}$ reorientation these two groups will be diametrically opposed and result in a diametrically bimodal distribution.

An alternative hypothesis, originally proposed by Cove (2000) and Fowler and Quatrano (1997), requires a decoupling of both the orientation, defined as the axis along which a zygote polarizes, and the sense of the polarizing vector, the position where the rhizoid is formed. This hypothesis would result in a fraction of zygotes with negative photopolarization, i.e., zygotes that polarize toward the illuminated instead of the shaded side relative to the first light cue (Figure 1, Situation II). As an example, if the fixation of the sense of the polarization vector lags behind the fixation of its orientation we expect a fraction of zygotes to have aligned both sense and direction to the L1 light vector, however another fraction that fixed the direction only according to L1 will find itself confused in the situation of L2 illumination. Here we expect about half will eventually polarize toward the L1 light source, while the other half develops its rhizoid away from the first light vector in addition to the previously mentioned fraction. When reorienting $180^{\circ}$, the fixation of the direction is irrelevant as it is the same for both light directions and will result again in a diametrical bimodal distribution. The fraction with negative photopolarization will not be discernible from the fraction that polarizes according to L2. At none of the time points 


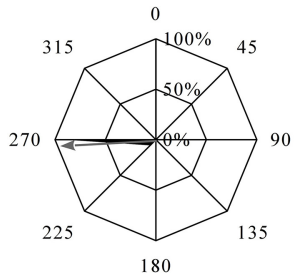

7h AF



11h AF



$13 \mathrm{~h} 00 \mathrm{AF}$

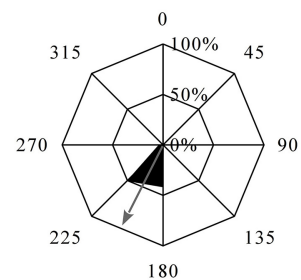

$15 \mathrm{~h} 00 \mathrm{AF}$

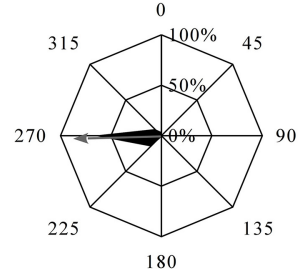

8h AF

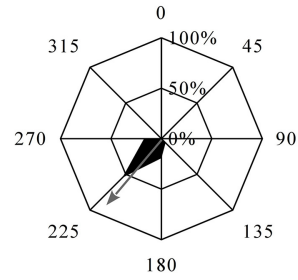

$11 \mathrm{~h} 30 \mathrm{AF}$

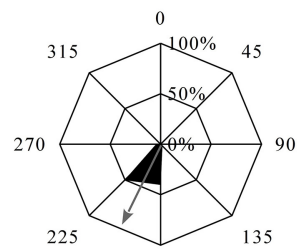

180

$13 \mathrm{~h} 30 \mathrm{AF}$

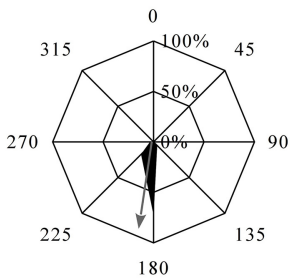

$16 \mathrm{~h} 00 \mathrm{AF}$



10h AF

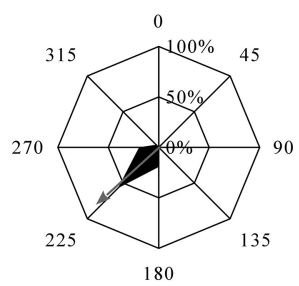

$12 \mathrm{~h} 00 \mathrm{AF}$

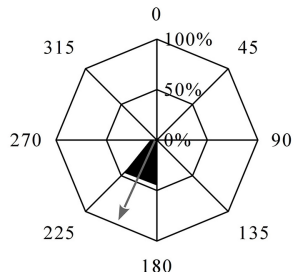

14h00 AF



17h00 AF

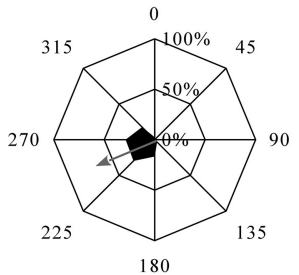

10h30 AF

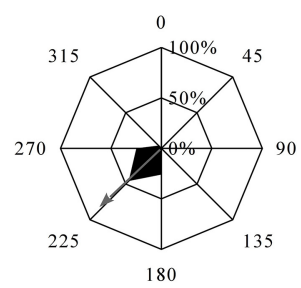

$12 \mathrm{~h} 30 \mathrm{AF}$

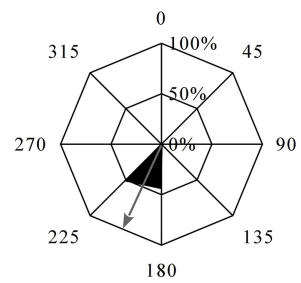

$14 \mathrm{~h} 30 \mathrm{AF}$

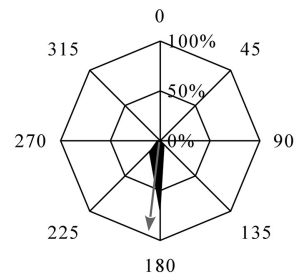

$18 \mathrm{~h} 00 \mathrm{AF}$
FIGURE 2 | Influence of $90^{\circ}$ reorientation experiments on the direction and sense of polarization vector. Second order mean of the direction and sense of the polarization vector after $90^{\circ}$ reorientation is depicted by an arrow. Length of the arrow represents the $r$ measure of the second order mean angle. Percentages represent the fraction of the population that shows a particular angular orientation of rhizoid outgrowth relative to the center of the cell. Angles represent the relative orientation of the rhizoid relative to the orientation of the first unidirectional light source. All mean angles of polarization vectors, including sense information, were statistically significant (df $=3, P<0.05)$. of reorientation an over-representation of the fraction with fixed polarization direction but misaligned polarization sense (relative to the first light vector) could be observed. Our experiments exclude the possibility that the cell fixes the direction before the sense of the polarization vector.

Both scenarios imply the complete breakdown of the old axis that is "forgotten" in favor of the de novo assembly of a new one. In other words the last axis amplification vector is assumed to determine the axis stabilization axis, while the old axis amplification vector is disassembled and has no influence on the future rhizoid site.

Alternatively, the initial polarization vector (including F-actin patch) may be disassembled, but not without leaving a trace or "imprint" in the cell or cell wall (Figure 1, Situation III). The information provided of multiple sequential light cues will then be integrated at the time of axis fixation and result in an intermediate angle, if oriented with $90^{\circ}$. When reoriented $180^{\circ}$ the light cues are diametrically opposed. Therefore, they will outweigh each other and the resultant angle will be according to the light cue that happens to be the strongest one and the distribution will be diametrically bimodal again. It is important to note that simultaneous fixation of direction and sense of the polarization vector is inherent to this scenario.

Indeed, we observed a different pattern than predicted by the two first scenarios. When reorienting $90^{\circ}$, zygotes show intermediate angles, which gradually rotate with increasing L1 
illumination time until the polarization axis is completely aligned with L1. The imprints of the polarization vectors following the perpendicular light vectors L1 and L2 (in terms of asymmetrical distribution of intrinsic determinants) will be added up and result in an intermediate angle (Figure 7). The longer the exposure to $\mathrm{L} 2$, the stronger the asymmetrical imprint of intrinsic determinants and the larger the influence of the L2 light vector will be at the moment of fixation. The length of each axis amplification vector in Figure 7 is proportional to this influence and the length of the illumination time within a certain time frame, roughly between 9 and $14 \mathrm{~h} \mathrm{AF}$, after which the axes are integrated and fixed. This period is much likely preceded with a period during

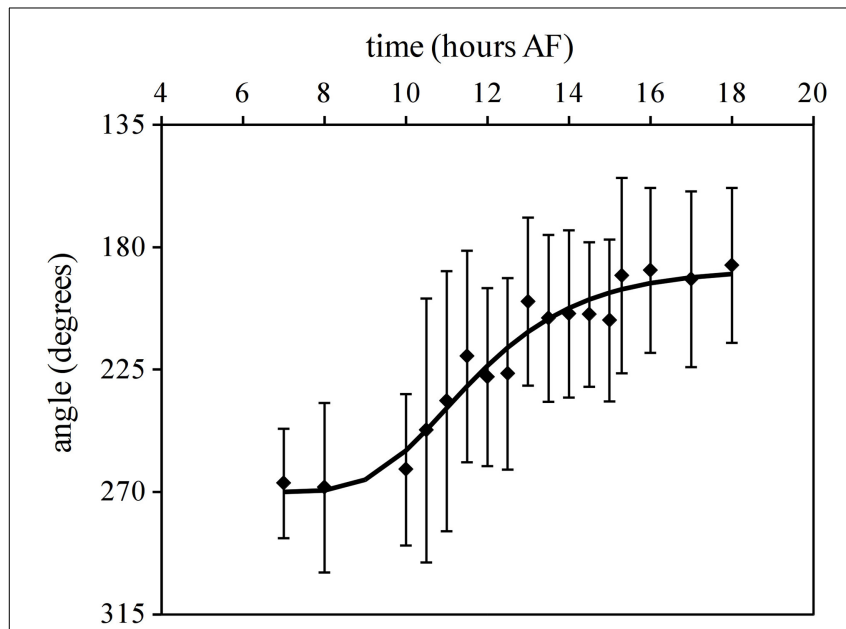

FIGURE 3 | Influence of reorientation on the fixation of direction and sense of polarization vector. Second order mean angles for the polarization vector after $90^{\circ}$ reorientation. Upper and lower error bars are Mardia's circular standard deviation for the mean angles $(n>200$ in three replicate experiments). The line depicts the regressed Gompertz sigmoid that best describes the data. which the cells can sense a light cue but are not able to imprint it in the cell wall yet. This is suggested by the fact a large fraction of the cells are already photoresponsive at 1-3 h AF in Fucus distichus (Kropf et al., 1989) at which time point the cells do not have secreted a uniform adhesive layer yet. It is important to note that simultaneous fixation of direction and sense is inherent to this scenario.

Under $180^{\circ}$ reorientation, the imprinting model implies a different interpretation of the point at which half of the cells commit to the first light vector compared to the previously discussed models. Traditionally this point is interpreted as a marker for axis fixation and denotes the point at which half of the cells have fixed their polarization axis and are therefore not sensitive any more to the second light vector (Quatrano, 1973; Kropf, 1989; Quatrano and Shaw, 1997). Under the imprinting hypothesis this is the point at which both light vectors equally influence the fixation of the polarization axis. Therefore, the imprinting model implies that the moment of axis fixation, assayed by TBO staining, occurs with slightly different kinetics compared to the commitment to the L1 vector (Figure 5). Indeed the data suggest half of the zygotes of the same three populations has an asymmetric TBO staining pattern only after $13.5 \mathrm{~h}$, which indeed coincides roughly with the end of the responsive period. Two hours earlier (10 h AF) $50 \%$ of zygotes already have determined the side of rhizoid formation as determined by L1. In Fucus distichus, however, it has been reported the moment of $50 \% \mathrm{~L} 1$ commitment and $50 \%$ of TBO staining coincided by $10 \mathrm{~h}$ AF (Shaw and Quatrano, 1996a,b) which contradicts our results. In contrast to the previous analysis, we report TBO staining at more than one time point and in more than one replicate population. Particularly the increased temporal resolution in the estimation of the F2 deposition kinetics might explain the apparent contradiction.

The different interpretation of the commitment to L1 in the 'imprinting model' links up with the controversy whether there exists an axis fixation event that is temporally distinct from germination (Quatrano, 1973) or whether the polarization axis

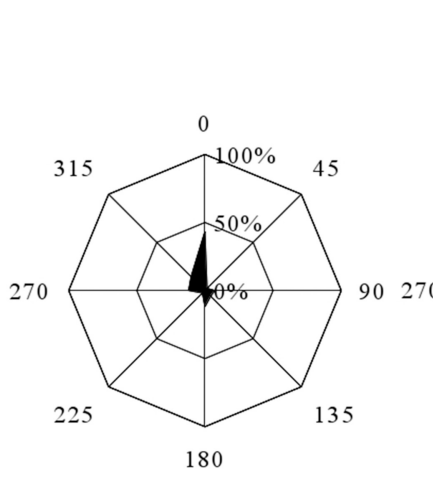

$10 \mathrm{~h} 30 \mathrm{AF}$


180

$12 \mathrm{~h} 00 \mathrm{AF}$

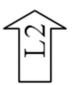

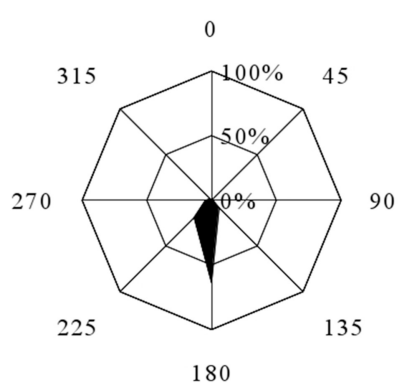

$16 \mathrm{~h} 30 \mathrm{AF}$
FIGURE 4 | Influence of $180^{\circ}$ reorientation experiment on the polarization vector for the time points $10.5,12$, and $16.5 \mathrm{~h}$ AF. Percentages aligned vertically represent the percentages of the population that show a particular angular orientation of rhizoid outgrowth relative to the center of the cell. Angles represent the relative orientation of the rhizoid relative to the orientation of the first unidirectional light source $(n=300$ in three replicate experiments). 
becomes fixed by local F2 deposition at the moment of germination itself as outlined by Robinson et al. (1999). While it is accepted that the axis is fixed before germination (Goodner and Quatrano, 1993), experiments involving an osmotic block of germination and subsequent repolarization might suggest there is no axis fixation before germination (Jaffe, 1990; Robinson, 1996).

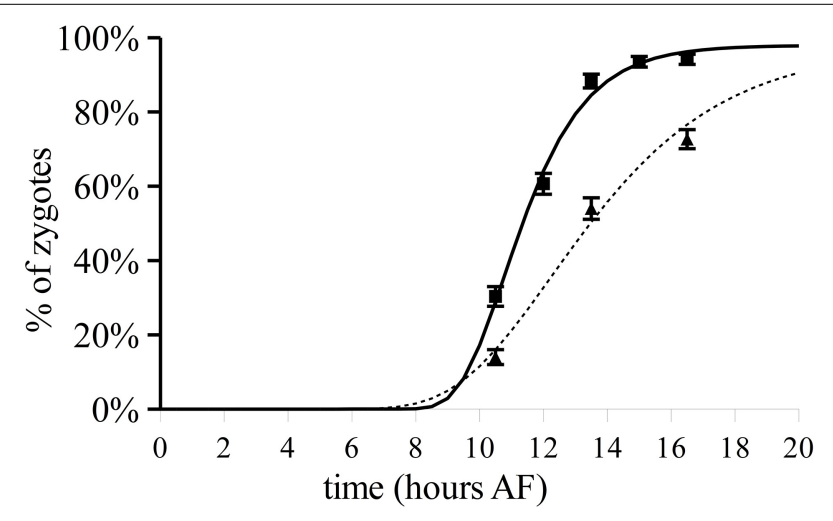

FIGURE 5 | Polarization after varying $180^{\circ}$ reorientation over time and TBO staining in zygotes developing under unchanged unilateral light. Fraction of zygotes having a polarization vector that points toward the shaded side in relation to L1 (squares). Percentage of zygotes staining asymmetrically with TBO under unilateral light (triangles). Upper and lower error bars are standard deviations ( $n=300$ in three replicate experiments). The lines depict the regressed Gompertz sigmoids that best describes the data (continuous, photopolarization according to L1; dotted, TBO patch staining).
Therefore, the commitment to the first light vector prior to polar growth as observed by Quatrano (1973), should have a different explanation than axis fixation. This explanation can be provided by the proposed imprinting model. The here-presented scenario implies that axis fixation occurs later than previously assumed based on reorientation data. If somehow the fixation of the cells is postponed (e.g., by osmotic block of polar growth), the L2 light source will still be able to reverse the polarization axis (Robinson et al., 1999).

It has been pointed out that the rapid fixation of a new polarization axis after reorientation is interesting and the fact that the axis remains labile for a long time is considered surprising (Kropf et al., 1999). Indeed one may expect $a$ priori that the process of amplification should occur with a constant speed. The long lability of the axis is surprising as it is difficult to imagine a distinct advantage in the intertidal. The moment the cells adhere at around 4-6 h AF one may expect a faster development would be possible and advantageous. This period of sensing the environment can be better understood in the context of the imprinting model as a period of averaging all environmental cues the cell perceives during a 5 -h interval. Fucoid zygotes in the intertidal rarely undergo continuous unilateral light as the sun migrates during this interval. Instead of betting on the last perceived vector, cells seem to average the perceived vector. This results in a polarization vector much more representative of the environment to which they need to adapt their development.

In conclusion, the here presented data together with the discussed published data can best be explained by the vectorial addition of imprints made during a large fraction of the cell cycle. How the information of the first environmental cue is stored, is

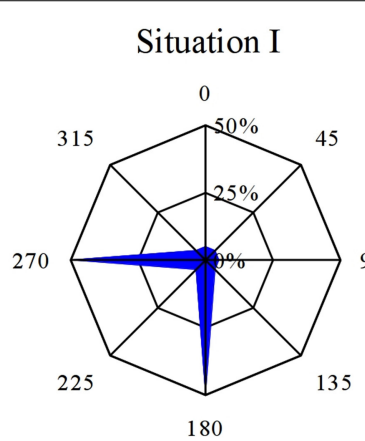

Situation I

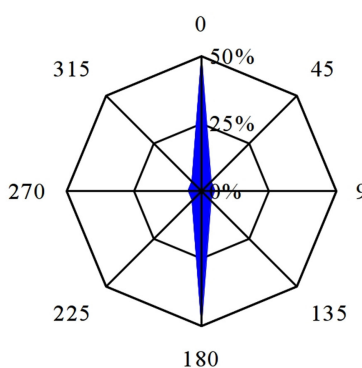

Situation II

0

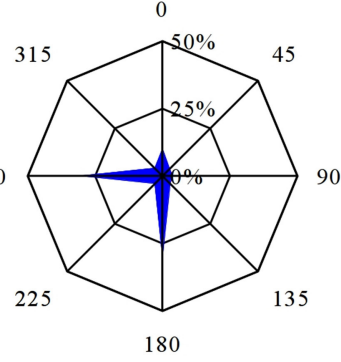

Situation II



Situation III

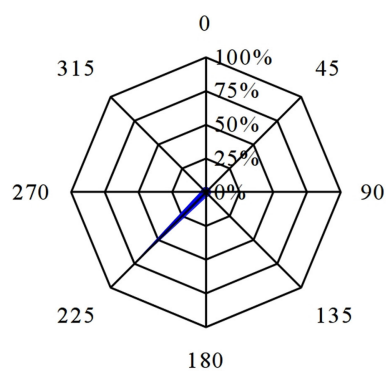

Situation III

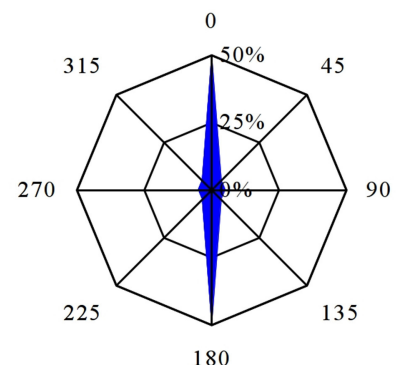

FIGURE 6 | Resultant angular distribution for each of the different polarization scenarios considered. Note that $180^{\circ}$ reorientation yields for each of these situations a diametrically bimodal distribution (see text for explanation). 




FIGURE 7 | Proposed scenario for integration of polarization vectors as vectorial sum under after reorientation of $90^{\circ}(\mathrm{A})$ or $180^{\circ}$ (B). AV, axis amplification vector; SV, axis stabilization vector. Red block arcs depict the hypothesized intrinsic factor responsible for the imprinting of unknown nature. Blue shading denotes F2 deposition as assayed by TBO staining. Hours denote duration of illumination under different light regimes and therefore the strength of each vector. Black bars and spheres denote the polar endomembrane system and F-actin deposition symbolizing the entire axis amplification machinery. not known. One can only speculate on the molecular nature of the cellular memory. Because the extracellular matrix has been shown to be an important way of storing developmental information controlling the cell fate (Bogaert et al., 2013), this part of the cell is the best location to search for the postulated intrinsic factor.

While fucoid zygotes are responsive to an impressive range of informational cues and develop in a dynamic environment with cues that may change from direction, cells normally develop only one rhizoid suggesting that multiple cues are integrated to generate a single one. While the axis amplification on itself is relatively well-documented, the way these cues are integrated is only circumstantially known (Hable and Hart, 2010). We have provided a better understanding in the way zygotes integrate sequentially applied vectors. How zygotes respond to simultaneously applied cues is still poorly understood. All cues that induce a signal are believed to be integrated in the common pathway upstream of establishment of the axis amplification (Kropf et al., 1999). But at which point their signaling cascade converges with each others ones is unknown. One may propose the here proposed imprinting mechanism would provide an alternative mechanism. But the fact that cells have only one amplification vector at the same time suggests this simultaneous integration occurs at the level of polarity signaling as proposed by Kropf et al. (1999).

\section{ACKNOWLEDGMENTS}

The authors are indebted to the Reasearch Foundation Flanders (FWO) (PhD fellowship to Kenny A. Bogaert). We thank Darryl
L. Kropf, David Smyth and Isabel Bäurle for helpful discussions and critical evaluation of the manuscript.

\section{SUPPLEMENTARY MATERIAL}

The Supplementary Material for this article can be found online at: http://www.frontiersin.org/journal/10.3389/fpls.2015. 00026/abstract

\section{REFERENCES}

Alessa, L., and Kropf, D. L. (1999). F-actin marks the rhizoid pole in living Pelvetia compressa zygotes. Development 126, 201-209.

Batschelet, E. (1972). "Recent statistical methods for orientation data," in Animal Orientation and Navigation, eds S. R. Galler, K. Schmidt-Koenig, G. J. Jacobs and R. E. Belleview (Washington D.C.: NASA), 61.

Batschelet, E. (1978). "Second-order statistical analysis of directions," in Animal migration, Navigation, and Homing, eds K. Schmidt-Koenig and W. T. Keeton (Berlin: Springer-Verlag), 1-24.

Belanger, K. D., and Quatrano, R. S. (2000). Polarity: the role of localized secretion. Curr. Opin. Plant Biol. 3, 67-72. doi: 10.1016/S1369-5266(99) 00043-6

Bisgrove, S. R. (2007). Cytoskeleton and early development in fucoid algae. J. Integr. Plant Biol. 49, 1192-1198. doi: 10.1111/j.1672-9072.2007.00518.x

Bogaert, K. A., Arun, A., Coelho, S. M., and De Clerck, O. (2013). Brown algae as a model for plant organogenesis. Methods Mol. Biol. 959, 97-125. doi: 10.1007/978-1-62703-221-6_6

Cove, D. J. (2000). The generation and modification of cell polarity. J. Exp. Bot. 51, 831-838. doi: 10.1093/jexbot/51.346.831

Fowler, J. E., and Quatrano, R. S. (1997). Plant cell morphogenesis: plasma membrane interactions with the cytoskeleton and cell wall. Annu. Rev. Cell Dev. Biol. $13,697-743$.

Fowler, J., and Quatrano, R. (1995). Cell polarity, asymmetric division, and cell fate determination in brown algal zygotes. Semin. Dev. Biol. 6, 347-358. 
Goodner, B., and Quatrano, R. S. (1993). Fucus embryogenesis: a model to study the establishment of polarity. Plant Cell 5, 1471-1481.

Hable, W. E. (2014). Racl signaling in the establishment of the fucoid algal body plan. Front. Plant Sci. 5:e00690. doi: 10.3389/fpls.2014.00690

Hable, W. E., and Hart, P. E. (2010). Signaling mechanisms in the establishment of plant and fucoid algal polarity. Mol. Reprod. Dev. 77, 751-758. doi: $10.1002 / \mathrm{mrd} .21199$

Hable, W. E., and Kropf, D. L. (2000). Sperm entry induces polarity in fucoid zygotes. Development 127, 493-501. doi: 10.1016/S1369-5266(00)80043-6

Hable, W. E., Reddy, S., and Julien, L. (2008). The Racl inhibitor, NSC23766, depolarizes adhesive secretion, endomembrane cycling, and tip growth in the fucoid alga, Silvetia compressa. Planta 227, 991-1000. doi: 10.1007/s00425-007-0673-1

Hadley, R., Hable, W. E., and Kropf, D. L. (2006). Polarization of the endomembrane system is an early event in fucoid zygote development. BMC Plant Biol. 6:5. doi: 10.1186/1471-2229-6-5

Hogsett, W., and Quatrano, R. (1978). Sulfation of fucoidin in Fucus embryos: III. Required for localization in the rhizoid wall. J. Cell Biol. 78, 866-873.

Hotelling, H. (1931). The generalization of student's ratio. Ann. Math. Stat. 2, 360-378.

Hurd, A. M. (1920). Effect of unilateral monochromatic light and group orientation on the polarity of germinating Fucus spores. Bot. Gaz. 70, 25-50.

Jaffe, L. (1958). Tropistic responses of zygotes of the Fucaceae to polarized light. Exp. Cell Res. 299, 282-299.

Jaffe, L. F. (1990). "Calcium ion currents and gradients in fucoid eggs," in Calcium in Plant Growth and Development, Vol. 4, eds R. T. Leonard and P. K. Hepler (Rockville, MD: American Society of Plant Physiology), 120-126.

Kropf, D. (1989). Protein synthesis and morphogenesis are not tightly linked during embryogenesis in Fucus. Dev. Biol. 134, 452-461.

Kropf, D. (1997). Induction of polarity in fucoid zygotes. Plant Cell 9, 1011-1020.

Kropf, D., Berge, S., and Quatrano, R. (1989). Actin localization during Fucus embryogenesis. Plant Cell 1, 191.

Kropf, D., Bisgrove, S., and Hable, W. (1999). Establishing a growth axis in fucoid algae. Trends Plant Sci. 4, 490-494.

Nuccitelli, R. (1978). Oöplasmic segregation and secretion in the Pelvetia egg is accompanied by a membrane-generated electrical current. Dev. Biol. 62, 13-33.

Peters, N. T., and Kropf, D. L. (2010). Asymmetric microtubule arrays organize the endoplasmic reticulum during polarity establishment in the brown alga Silvetia compressa. Cytoskeleton 67, 102-111. doi: 10.1002/cm.20427

Quatrano, R., and Crayton, M. (1973). Sulfation of fucoidan in Fucus embryos: I. Possible role in localization. Dev. Biol. 30, 29-41.
Quatrano, R. S. (1973). Separation of processes associated with differentiation of two-celled Fucus embryos. Dev. Biol. 30, 209-213.

Quatrano, R., and Shaw, S. (1997). Role of the cell wall in the determination of cell polarity and the plane of cell division in embryos. Trends Plant Sci. 2, 15-21.

Robinson, K. (1996). Calcium and the photopolarization of Pelvetia zygotes. Planta 198, 378-384.

Robinson, K., Wozniak, M., Pu, R., and Messerli, M. (1999). Symmetry breaking in the zygotes of the fucoid algae: controversies and recent progress. Curr. Top. Dev. Biol. 44, 101-125.

Schröter, K. (1978). Asymmetrical jelly secretion of zygotes of Pelvetia and Fucus: an early polarization event. Planta 140, 69-73.

Shaw, S. L., and Quatrano, R. S. (1996a). Polar localization of a dihydropyridine receptor on living Fucus zygotes. J. Cell Sci. 109, 335-342.

Shaw, S. L., and Quatrano, R. S. (1996b). The role of targeted secretion in the establishment of cell polarity and the orientation of the division plane in Fucus zygotes. Development 122, 2623-2630.

Ueda, M., and Laux, T. (2012). The origin of the plant body axis. Curr. Opin. Plant Biol, 15, 578-584. doi: 10.1016/j.pbi.2012.08.001

Vreeland, V., Grotkopp, E., Espinosa, S., Quiroz, D., Laetsch, W. M., and West, J. (1993). The pattern of cell wall adhesive formation by Fucus zygotes. Hydrobiologia 260-261, 485-491.

Conflict of Interest Statement: The authors declare that the research was conducted in the absence of any commercial or financial relationships that could be construed as a potential conflict of interest.

Received: 13 November 2014; paper pending published: 12 December 2014; accepted: 12 January 2015; published online: 03 February 2015.

Citation: Bogaert KA, Beeckman T and De Clerck O (2015) Photopolarization of Fucus zygotes is determined by time sensitive vectorial addition of environmental cues during axis amplification. Front. Plant Sci. 6:26. doi: 10.3389/fpls.2015.00026

This article was submitted to Plant Evolution and Development, a section of the journal Frontiers in Plant Science.

Copyright (C) 2015 Bogaert, Beeckman and De Clerck. This is an open-access article distributed under the terms of the Creative Commons Attribution License (CC BY). The use, distribution or reproduction in other forums is permitted, provided the original author(s) or licensor are credited and that the original publication in this journal is cited, in accordance with accepted academic practice. No use, distribution or reproduction is permitted which does not comply with these terms. 